\title{
Dynamic effects of the Nuss procedure on the spine in asymmetric pectus excavatum
}

\author{
Tomohisa Nagasao, MD, PhD, ${ }^{\mathrm{a}}$ Masahiko Noguchi, $\mathrm{MD}, \mathrm{PhD},{ }^{\mathrm{b}}$ Junpei Miyamoto, $\mathrm{MD}, \mathrm{PhD},{ }^{\mathrm{a}}$ \\ Hua Jiang, MD, PhD, ${ }^{\mathrm{c}}$ Weijin Ding, $\mathrm{MD},{ }^{\mathrm{c}}$ Yusuke Shimizu, MD, ${ }^{\mathrm{a}}$ and Kazuo Kishi, MD, PhD ${ }^{\mathrm{a}}$
}

Objective: This study aimed to elucidate dynamic effects of the Nuss procedure on the spine in the treatment of patients with pectus excavatum with asymmetric thoraces.

\begin{abstract}
Methods: Twenty-five patients with pectus excavatum who underwent the Nuss procedure were categorized into 4 groups by preoperative morphology of the spine and thoracic asymmetry. In group $1(n=8)$, the right side of the thorax was concave and the spine bowed to the right. In group $2(n=4)$, the right side of the thorax was concave and the spine bowed to the left. In group $3(n=5)$, the left side of the thorax was concave and the spine bowed to the right. In group $4(n=8)$, the left side of the thorax was concave and the spine bowed to the left. With computed tomographic data, finite-element models were produced to simulate each patient's thorax. Thereafter, dynamic response patterns of the spine to the Nuss procedure were examined. Validity of these biomechanical findings was verified by referring to clinical outcomes.
\end{abstract}

Results: In group 1 and group 4 models, deformed spines were straightened; in group 2 and group 3 models, spinal bowing increased. These biomechanical findings were compatible with clinical evaluations.

Conclusions: Performance of the Nuss procedure for asymmetric pectus excavatum exerts dynamic influence on the spine. Response patterns of the spine are predictable from morphologic relationships between the asymmetric patterns of the anterior thoracic wall and the spine. (J Thorac Cardiovasc Surg 2010;140:1294-9)

F Supplemental material is available online.

Although attention is usually paid only to the anterior part of the thorax in the correction of pectus excavatum, we have focused on the posterior part of the thorax-the spine. The Nuss procedure aims to correct concavity of the anterior region of the thorax through the placement of bars. The dynamic effect of the bar placement is not necessarily restricted to the anterior region, however, because the anterior and posterior regions of the thorax are not independent entities, a dynamic event occurring in one can affect the other. Development of scoliosis has been reported ${ }^{1}$ as a complication involving the spine after the Nuss procedure. To avoid

From the Department of Plastic and Reconstructive Surgery, ${ }^{a}$ School of Medicine, Keio University, Tokyo, Japan; the Department of Plastic Surgery, ${ }^{\mathrm{b}}$ Nagano Children's Hospital, Azumino, Japan; and the Department of Plastic Surgery, ${ }^{\mathrm{c}}$ Shanghai Second Military Medical College, ChangZheng Hospital, Shanghai, China.

Supported in part by a Grant-in-Aid for Scientific Research from the Ministry of Education, Culture, Sports, Science and Technology of Japan (C-21500453).

Disclosures: None.

Received for publication March 13, 2010; revisions received May 17, 2010; accepted for publication June 20, 2010; available ahead of print July 22, 2010.

Address for reprints: Tomohisa Nagasao, MD, PhD, Department of Plastic and Reconstructive Surgery, Keio University Hospital, Shinjuku-Ward Shinanomachi 35, Tokyo Japan (E-mail: nagasao@sc.itc.keio.ac.jp).

$0022-5223 / \$ 36.00$

Copyright (c) 2010 by The American Association for Thoracic Surgery doi: 10.1016/j.jtcvs.2010.06.025 such complications, we need to understand how the spine responding to the Nuss procedure behaves. This study was aimed at elucidating this issue.

\section{MATERIALS AND METHODS \\ Study Sample}

From among patients with asymmetric pectus excavatum who underwent the Nuss procedure at our institutions from 2006 to 2010 , a total of 25 patients (14 male and 11 female) with asymmetry of the thorax and mild to moderate deformity of the spine (with Cobb angle of $\leq 30^{\circ}$ ) were randomly selected. The selection of patients was performed by a group consisting of 3 physicians ( 2 plastic surgeons [T.N. and J.M.] and 1 orthopedist) by referring to radiographic or computed tomographic (CT) images collected preoperatively. Correction bars were placed at the 4 th to 6 th intercostal spaces. Depending on the severity of the deformity, 1 to 3 correction bars were used for each patient.

The 25 patients were divided into the following 4 groups according to preoperative asymmetry patterns of the anterior thoracic wall and the spine. In group $1(\mathrm{n}=8)$, the anterior thoracic wall was concave on the right side and the spine bowed toward the right side. In group $2(n=4)$, the anterior thoracic wall was concave on the right side and the spine bowed toward the left side. In group $3(n=5)$, the anterior thoracic wall was concave on the left side and the spine bowed toward the right side. In group 4 $(n=8)$, the anterior thoracic wall was concave on the left side and the spine bowed toward the left side.

The patients ages were $12.2 \pm 5.3$ years old in group $1,11.5 \pm 1.9$ years old in group 2; $9.6 \pm 2.7$ years old in group 3, and $12.4 \pm 3.3$ years old in group 4. Patients' ages demonstrated no statistically significant differences between any 2 groups.

Cobb angles were $23.2^{\circ} \pm 5.8^{\circ}$ in group $1,22.7^{\circ} \pm 3.4^{\circ}$ in group 2 , $24.0^{\circ} \pm 4.5^{\circ}$ in group 3 , and $21.1^{\circ} \pm 5.2^{\circ}$ in group 4 . Cobb angles demonstrated no statistically significant differences between any 2 groups.

Haller indices were $5.4 \pm 2.3$ for group $1,4.9 \pm 2.3$ for group 2, $5.7 \pm$ 3.0 for group 3 , and $5.5 \pm 2.2$ for group 4 . Asymmetry indices (the value obtained by dividing the anteroposterior thorax length of the nonconcave 


\section{Abbreviations and Acronyms \\ $\% \mathrm{VC}=$ percentage of predicted vital capacity \\ $\mathrm{CT}=$ computed tomography}

side with that of the concave side ${ }^{2}$ ) were $0.72 \pm 0.06$ for group $1,0.73 \pm$ 0.10 for group 2, $0.72 \pm 0.09$ for group 3, and $0.71 \pm 0.07$ for group 4 . Both Haller indices and asymmetry indices demonstrated no statistically significant differences between any 2 groups.

\section{Biomechanical Model Study}

Model production. For each patient, data for the thorax region were extracted from the corresponding CT data with graphic software (Rhinoceros 4.0; Applicraft Co, Tokyo, Japan). The data were further edited with structural analysis software (ANSYS11.0; ANSYS Co, Chicago, Ill), to produce a finite-element analysis model for each thorax (Figure E1). ${ }^{3,4}$ The 12 ribs, sternum, and 12 vertebrae were each modeled with 6-, 18-, and 36beam elements, respectively in the simulations. Young's moduli were calculated from the CT density of each component according to the equation of Kopperdahl and colleagues ${ }^{5}: \mathrm{E}=-34.7+3230 \cdot$ QCT, where $E$ and $Q C T$ are, respectively, the Young's modulus in megapascals and the CT density in grams per milliliter. Young's moduli were allotted to each component of the thorax: 1460 to $2020 \mathrm{MP}$ (mean, $1740 \mathrm{MP}$ ) for cortical bone, 154 to 206 (mean, 180 MP) for cancellous bone, and 62 to 110 (mean, 82MP) for costal cartilage. Simulation models corresponding to the 4 groups defined in the previous subsection are shown in Figure 1.

Load application to simulate the Nuss procedure. With the models produced, the Nuss procedure was simulated by elevating the sternum until the concavity of the anterior region of the thorax was corrected (Figure 2). Assuming that correction bars contacted the posterior aspect of the sternum $(R$ in Figure 2$)$ and were supported at the costochondral junctions ( $P$ and $Q$ in Figure 2) of the corresponding intercostal spaces, loads were applied on these points until the posterior aspect of the sternum $(R)$ reached the segment connecting bilateral costochondral junctions $(P$ and $Q)$. Because the bars were placed at varying intercostal spaces in actual operations, the loading was conducted at the corresponding intercostal spaces for each patient. Accordingly, for patients in whom multiple bars were placed, the load application was performed at multiple intercostal spaces.

Evaluation of spine shape. The changes in the spinal shape before and after loading were evaluated. When the spine was twisted in the direction of its original bowing, the change was evaluated as deterioration; when the spine was twisted in the opposite direction from its original bowing, the change was evaluated as improvement. For instance, in spines of the thoraces belonging to group 1 patients-in whom the spine bowed toward the right side- the shape of the spine was evaluated to have improved if it was twisted toward the left side.

\section{Evaluation of Clinical Outcomes}

Evaluation of perioperative spine shapes on radiographic images. The change in the degree of bending of the spine before and after the operation was evaluated for each patient by referring to preoperative and postoperative radiographic images of the thoracoabdominal regions. The preoperative radiographic images were taken within 1 month before the operation; the postoperative radiographic images were taken from 3 to 6 months after the operation. The evaluation was conducted by a board of 3 physicians -1 plastic surgeon (T.N.) and 2 orthopedists. Each change was classified into 1 of 3 ranks: improvement, no change, and deterioration. When the spine was straightened after the operation, the change was evaluated as improvement; when the spine showed no evident transformation, the change was evaluated as no change; and when the spine showed more serious distortion after the operation than it did preoperatively, the change was evaluated as deterioration. The Pearson $\chi^{2}$ test was used to compare differences in the distribution patterns of the evaluation results of groups 1 and 2 and groups 3 and 4 .

The curvature types of the spines preoperatively and postoperatively were evaluated by referring to King and colleagues' classification. ${ }^{6}$ For each of the 4 groups, patterns of change in types and their frequencies were evaluated.

Respiratory function. For each of the 4 groups, preoperative and postoperative respiratory function was compared with reference to each patient's percentage of predicted vital capacity $(\% \mathrm{VC}$, given as a percentage of the vital capacity predicted from calculation of the person's age, sex, and height, normally $\geq 80 \%$ ). The paired $t$ test was used for this comparison.

\section{Statistical Methods}

For statistical calculations, SPSS Version 10 for Windows (SPSS Inc, an IBM Company, Chicago, Ill) was used.

\section{RESULTS}

\section{Biomechanical Model Study}

Examples of the deformity patterns for the 4 groups are demonstrated in Figure 1. In group 1, with all models, the spine deviated to the left side. Because the spine had initially bowed toward the opposite side, the shape of the spine demonstrated improvement. In group 2, with all models, the spine deviated to the left side. Because the spine had initially bowed toward the same side, the shape of the spine demonstrated deterioration. In group 3, with all models, the spine deviated to the right side. Because the spine had initially bowed toward the same side, the shape of the spine demonstrated deterioration. In group 4, with all models, the spine deviated to the right side. Because the spine had initially bowed toward the opposite side, the shape of the spine demonstrated improvement.

\section{Evaluation of Clinical Results}

Degree of bending. For each group, distribution of the numbers of patients belonging to the 3 ranks is shown in Table 1. Examples of spinal transformation for two cases-with their preoperative conditions belonging to groups 2 and 3-are demonstrated in Figure 3. The following are the results of intergroup comparisons. Distribution of the 3 ranks was statistically significantly different between groups 1 and $2(P=.002)$. This finding indicates that the operation tended to straighten the spine for group 1 and to exacerbate the bowing of the spine for group 2. Distribution of the 3 ranks was statistically significantly different between groups 3 and $4(P=.033)$. This finding indicates that the operation tended to exacerbate the bowing of the spine for group 3 and to straighten the spine for group 4.

Curvature types. Change in curvature type is indicated as in the following example: T2-T1 (2) indicates that there were 2 cases with King and colleagues' type 2 deformity preoperatively and type 1 deformity postoperatively. The following results were obtained: in group 1 (8 cases), T2T1 (1), T3-T3 (4); T4-T3 (1), and T5-T1 (2); in group 2 (4 cases), T3-T2 (3) and T3-T4 (1), in group 3 (5 cases), 


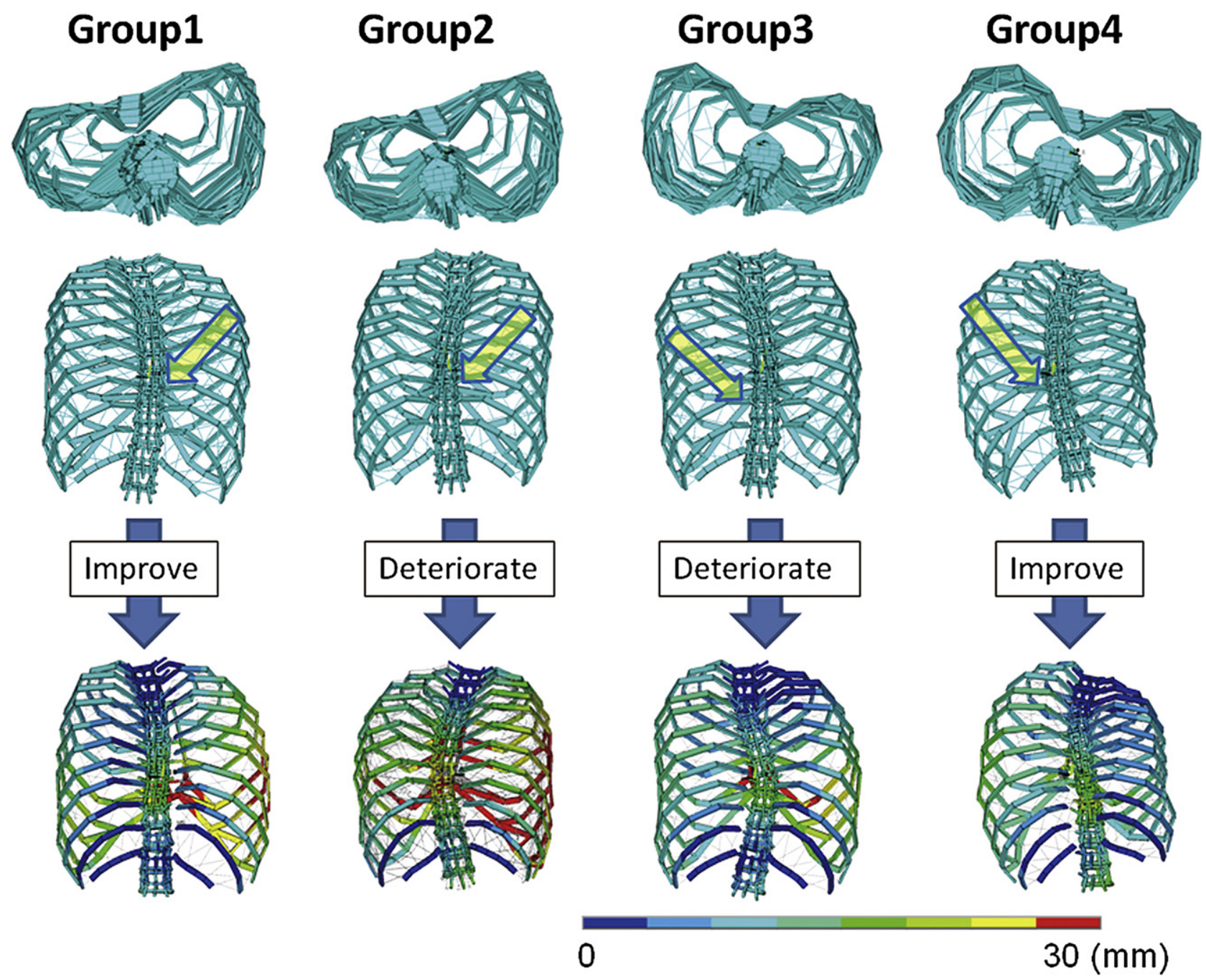

FIGURE 1. Examples of models for thoraces belonging 4 morphologic groups and their transformation patterns in Nuss procedure simulation. Upper row shows representative thorax models for groups 1 to 4, viewed from below. Middle row shows representative thorax models for groups 1 to 4 , viewed from behind. As correction bars are placed, thoraces receive counterforces. Direction of counter forces differs according to preoperative deformity patterns of anterior thoracic wall (yellow arrows). Lower row shows transformation patterns of models responding to load application simulating Nuss procedure. Color scale indicates degree of deviation.

T2-T2 (1), T3-T2 (1), T3-T4 (2), and T3-T5 (1); in group 4 (8 cases), T2-T1 (1), T3-T3 (4), T4-T3 (2), and T4-T4 (1). Although about half the spines in groups 1 and 4 had no curvature type change, most of the spines in groups 2 and 3 had curvature type change.

Respiratory function. In groups 1 and 4 , the values of $\% \mathrm{VC}$ increased postoperatively. In groups 2 and 3, the values of $\% \mathrm{VC}$ did not demonstrate statistically significant differences in the preoperative versus postoperative comparison. The preoperative values of $\% \mathrm{VC}$ were $64.6 \% \pm 6.0 \%$ in group $1,64.3 \% \pm 5.8 \%$ in group $2,68.0 \% \pm 3.4 \%$ in group 3 , and $70.0 \% \pm 3.3 \%$ in group 4 . The postoperative values of $\% \mathrm{VC}$ were $73.3 \% \pm 8.1 \%$ in group $1,65.3 \% \pm$ $3.8 \%$ in group $2,70.0 \% \pm 4.0 \%$ in group 3 , and $81.1 \% \pm$ $2.6 \%$ in group 4 .

\section{DISCUSSION}

Because of its technical ease and reduced invasiveness, the Nuss procedure is among the most effective and frequently used surgical methods for the correction of pectus excavatum. ${ }^{7,8}$ Although the Nuss procedure was initially used for juvenile patients, ${ }^{9}$ application of the procedure has extended to include patients of a wider age range. ${ }^{10-12}$ In addition to pectus excavatum, a modified version of the Nuss procedure is also used for treatment of pectus carinatum. ${ }^{13}$ The main purpose of the Nuss procedure is to correct the anterior region of the thorax; however, we believe that in performing the Nuss procedure, attention should be paid not only to the anterior region of the thorax but also to the posterior region-more specifically, the spine-because pectus excavatum is often accompanied by deformity of the spine. ${ }^{14}$ 

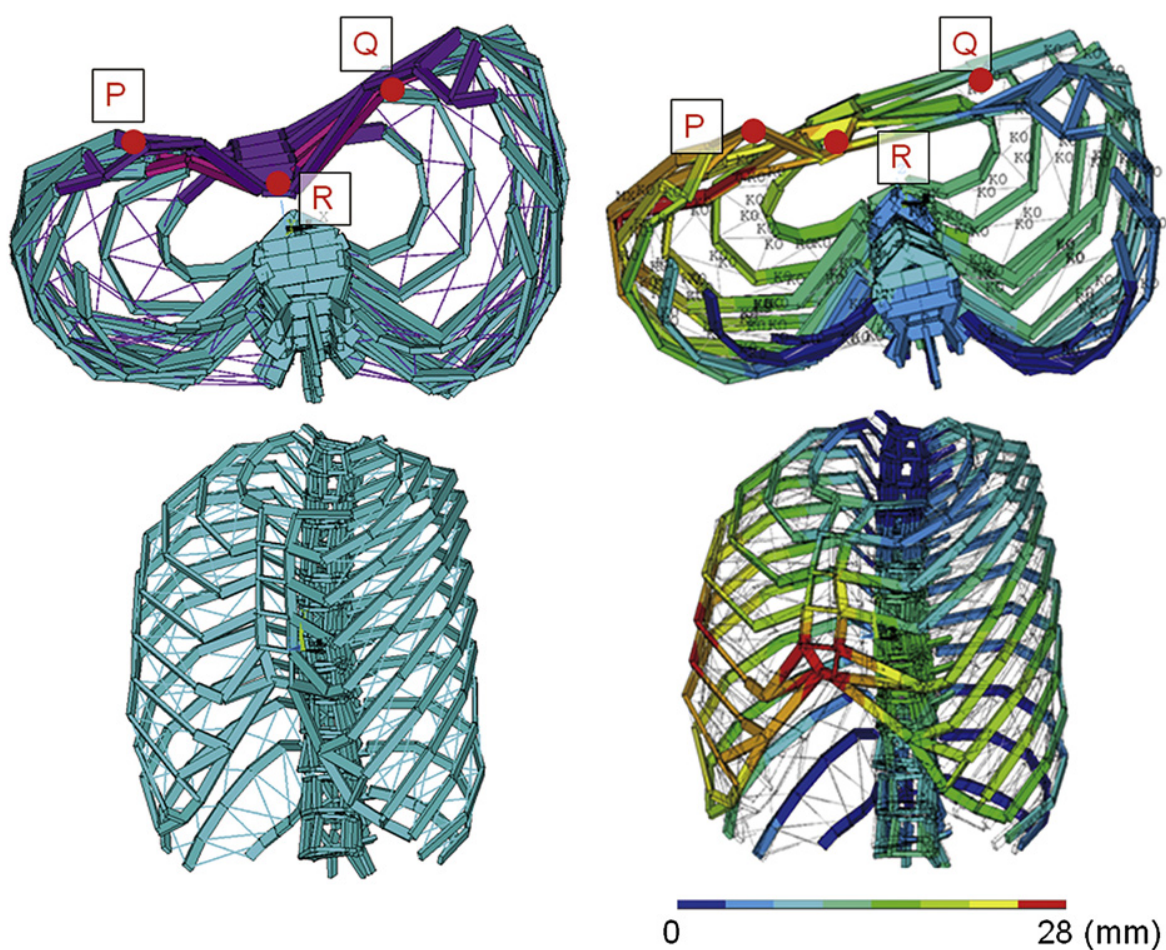

FIGURE 2. Simulation of Nuss procedure viewed from below (A) and obliquely (B). Contour indicates degree of deviation. $R$, posterior aspect of sternum; $P$ and $Q$, costochondral junctions.

We have conducted a theoretic experiment to simulate the Nuss procedure. An asymmetric thorax with severe concavity on the right side is assumed (Figure 4, top). To correct the concave deformity in the anterior region, the bar exerts an elevation force to the sternum ( $E F$ in Figure 4). Because of the asymmetry, the elevation takes place in the right oblique direction (note that Figure 4 is viewed from below). Accordingly, the elevation force is directed right obliquely. In response to this elevation, the thorax receives counterforces ( $R 1$ and $R 2$ ) from the correction bars. Because the counterforces work in the opposite direction of the elevation force, they work in the left oblique direction. The counterforces are transmitted to the spine and work to bow it in the left direction. The spine presents different response patterns to these forces depending on its preoperative condition. Spines that are bowed left preoperatively are straightened by the leftdirected counterforces (Figure 4, below left). Contrarily, in spines preoperatively bending right, distortion is exacerbated by the counterforces (Figure 4, below right). With

TABLE 1. Distribution of the evaluations of the spines' transformations

\begin{tabular}{lccc}
\hline & Improvement & No change & Deterioration \\
\hline Group 1 $(\mathrm{n}=8)$ & 8 & 0 & 0 \\
Group 2 $(\mathrm{n}=4)$ & 0 & 1 & 3 \\
Group 3 $(\mathrm{n}=5)$ & 0 & 1 & 4 \\
Group 4 $(\mathrm{n}=8)$ & 5 & 2 & 1 \\
\hline
\end{tabular}

thoraces with serious concavity on the left side, the spine takes contrary patterns to those presented in this example.

Reasoned this way, it is hypothesized that in correction of asymmetric pectus excavatum, the spine receives a force directed from the side of the anterior wall with concavity to the contralateral side, and the spine bends in this direction. To verify this hypothesis, we classified patients' thoraces into 4 patterns and conducted this study. We used 2 different approaches - the model study and evaluation of clinical results - to verify the hypothesis, thus verifying the hypothesis from both theoretic and practical standpoints.

In the model study, we used finite-element analysis. Finiteelement analysis is an established study method used for biomechanical analyses of various organs of the body. ${ }^{15,16}$ In all thoracic models in this study, the spine deviated from the side with serious anterior thoracic concavity to the contralateral side, which was compatible with our hypothesis.

In the evaluation of clinical results, we demonstrated that the shape of the spine improved for patients in groups 1 and 4 and deteriorated in groups 2 and 3 . This also supported the validity of the previously started hypothesis.

\section{CONCLUSIONS}

We conclude the following:

1. In the Nuss procedure for asymmetric pectus excavatum thoraces, the spine transforms in response to the placement of the bars. 

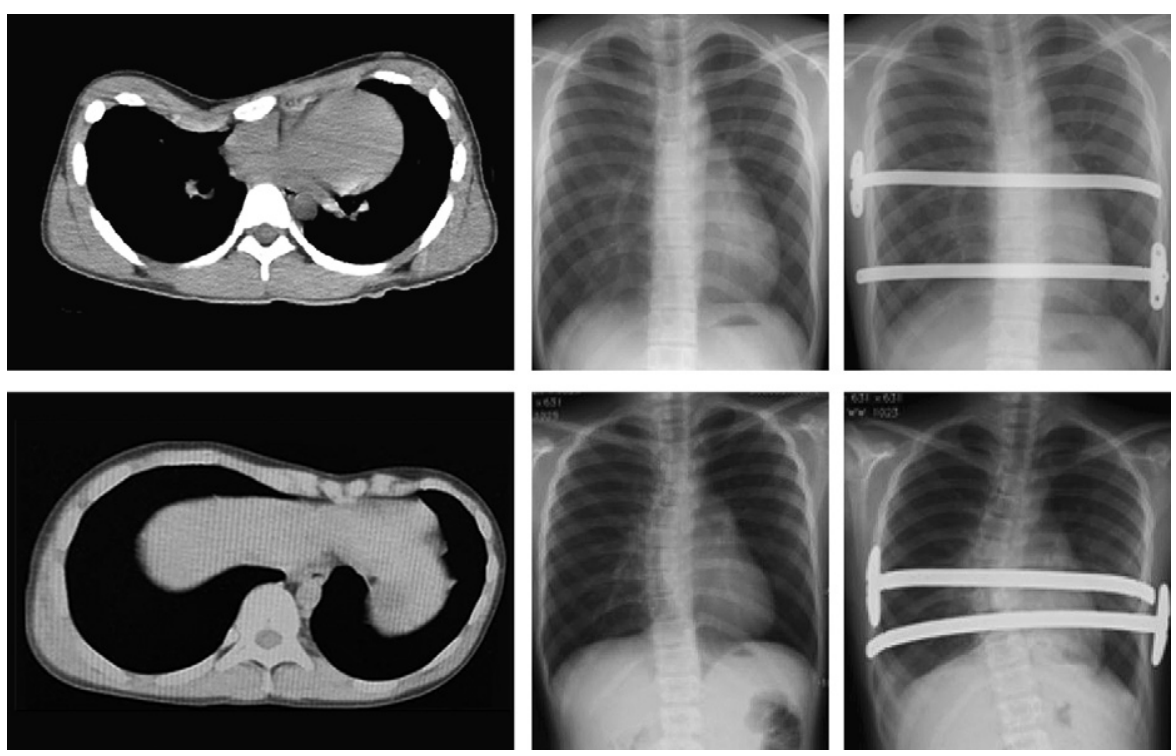

FIGURE 3. Upper left, Preoperative computed tomographic image of group 2 patient. Anterior thoracic wall was concave on right side. Upper center and upper right, Preoperative and postoperative radiographic images, respectively, of group 2 patient. After operation, spine bowed to left side. Lower left, Preoperative computed tomographic image of group 3 patient. Anterior thoracic wall was concave on left side. Lower center and lower right, Preoperative and postoperative radiographic images, respectively, of group 3 patient. Bowing of spine to right side increased after operation.

2. The transformation bends the spine from the side of serious concavity to the side with less concavity.

3. Whether or shape of the spine improves depends primarily on the preoperative morphologic relationship between the anterior region and spine. When the side of spinal bowing coincides with the side of anterior wall concavity preoperatively, the spine is straightened; when the spine bows to the opposite side of the anterior wall concavity preoperatively, the deformity of the spine increases.

These principles are clinically important, because they can be used in planning treatment strategy for patients with asymmetric pectus excavatum. In particular, care should be taken in planning the Nuss procedure for patients in group 2 or group 3, because application of the Nuss procedure could aggravate deformity of the spine for these patients (Figure E2).

The results of the respiratory function testing also indicate that special care should be taken for patients in groups 2 and 3 . Because the concavity of the anterior chest wall is corrected with the Nuss procedure, the volume of the thoracic space increases. Accordingly, the value of $\% \mathrm{VC}$ should increase. This expectation held true with the patients in groups 1 and 4 ; however, in groups 2 and 3 , the values of $\% \mathrm{VC} \mathrm{dem}$ onstrated no increase after operation. We speculate that for these patients aggravated distortion of the spine negatively works on respiratory movement and offsets the positive effect of the increase in thoracic volume. We further speculate that performance of the Nuss procedure for patients with serious spine distortion-although such patients are not included in this study-would even worsen respiratory function. In addition to distortion of the spine, respiratory function should therefore also be monitored when performing the Nuss procedure for patients in groups 2 and 3.

In terms of the spinal transformation, some patients demonstrated a discrepancy between the theoretic expectation
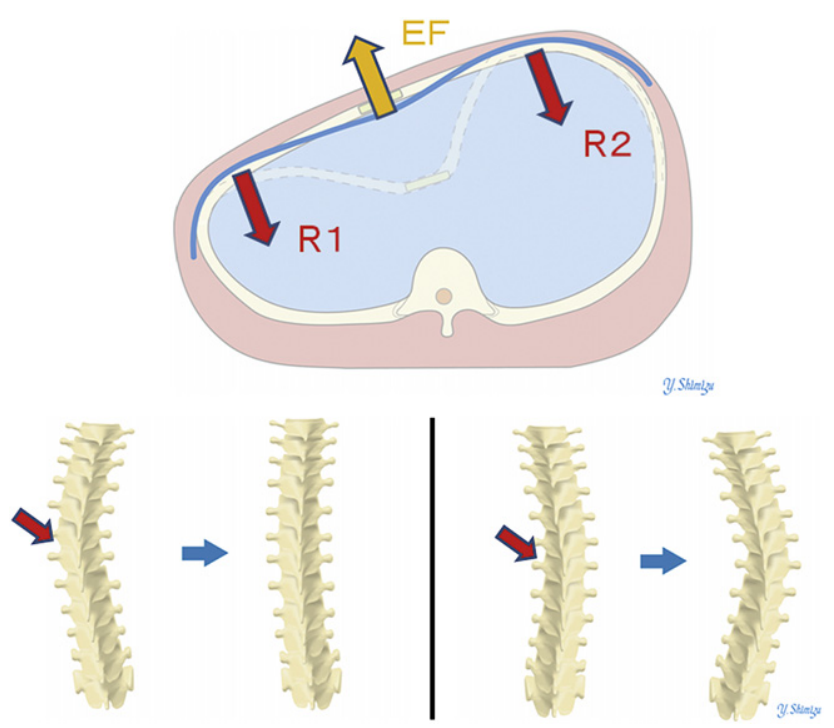

FIGURE 4. Top, Diagram of thorax viewed from below. $E F$ indicates force that bar exerts on sternum. $R 1$ and $R 2$ indicate counterforces that thorax receives from bar. Bottom, $R 1$ and $R 2$ are transmitted to spine and work to bend it toward left side. Bottom left, When spine bows to left side in preoperative condition, it is straightened by transmitted forces (red oblique arrow). Bottom right, When spine bows to right side preoperatively, it is further bent to right side, increasing deformity. 
and actual clinical outcome. For instance, although our hypothesis predicts all spines of group 3 patients to show exacerbated deformity after the operation, 1 of the 5 patients in that group showed no change. Contrarily, although spine shapes are theoretically expected to improve in group 4 patients, 1 of the 8 patients in that group showed deterioration. The discrepancy between the theory and actual results can be attributed to various complicating factors not accounted for in our simulation, such as the function of the erector spinal muscles. When the application of the correction bars works to increase the distortion of the spine, the erector spinal muscles diminish the effect by exerting counterforce to straighten the spine. On the other hand, even if the correction bars work to straighten the spine, the spine can show increased deformity if the spine-straightening effect produces pain and the patient develops a habit of twisting the body to reduce the pain.

The fact that the geometric types of spinal curvature change postoperatively also indicates that complicated factors affect spine shape. If the placement of the correction bars solely affected spine shapes, geometric type of the spine would be expected to show no change, although the degree of the curvature might change. Evaluation of the clinical outcomes, however, revealed shifting of the geometric types in a considerable percentage of cases. It is an interesting finding that the ratio of the geometric type change differed among groups. In groups 1 and 4, about half the patients showed no change in geometric type, whereas in groups 2 and 3 , most patients showed a type shift. We explain these phenomena through the compensation mechanism of the erector spinal muscles. In groups 1 and 4, the Nuss procedure works to straighten the spine. Because this is a good change, the compensation mechanism does not operate. Contrarily, in groups 2 and 3, the spine is distorted by the operation. In these situations, the erector muscles counteract to mitigate the unfavorable change. Receiving effects from the forces counteracting each other, the spine demonstrates a complicated deformity. In groups 2 and 3, the geometric types of the spine are likely to change for this reason.

In this study, evaluation of clinical outcomes was conducted by referring to radiographic images taken between 3 and 6 months postoperatively. The shapes of the spine could gradually change afterward, either in patients who develop habits of twisting the body or in those in whom the erector spinal muscles continue to exert counteraction.
Furthermore, patients with both pectus excavatum patients and serious scoliosis (Cobb angle $\geq 30^{\circ}$ ) were not included in this study, because we put higher priority on the treatment of scoliosis than on the correction of pectus excavatum in such cases. To strengthen the generality and validity of this study's findings, we plan to expand the study in the future by gathering larger samples and by performing followup for longer periods.

\section{References}

1. Niedbala A, Adams M, Boswell WC, Considine JM. Acquired thoracic scoliosis following minimally invasive repair of pectus excavatum. Am Surg. 2003;69: 530-3.

2. Cartoski MJ, Nuss D, Goretsky MJ, Proud VK, Croitoru DP, Gustin T, et al. Classification of the dysmorphology of pectus excavatum. J Pediatr Surg. 2006;41: 1573-81.

3. Nagasao T, Miyamoto J, Jiang H, Ichihara K, Tamaki T, Taguchi T, et al. Stress distribution on the thorax after the Nuss procedure for pectus excavatum results in different patterns between adult and child patients. $J$ Thorac Cardiovasc Surg. 2007;134:1502-7.

4. Nagasao T, Miyamoto J, Kokaji K, Yozu R, Jiang H, Jin H, et al. Double-bar application decreases postoperative pain after Nuss procedure. J Thorac Cardiovasc Surg. Epub 2010 Apr 2.

5. Kopperdahl DL, Pearlman JL, Keaveny TM. Biomechanical consequences of an isolated overload on the human vertebral body. J Orthop Res. 2000;18: 685-90.

6. King HA, Moe JH, Bradford DS, Winter RB. The selection of fusion levels in thoracic idiopathic scoliosis. J Bone Joint Surg Am. 1983;65:1302-13.

7. Park HJ, Jeong JY, Jo WM, Shin JS, Lee IS, Kim KT, et al. Minimally invasive repair of pectus excavatum: a novel morphology-tailored, patient-specific approach. J Thorac Cardiovasc Surg. 2010;139:379-86.

8. Coelho M, Silva RF, Bergonse Neto N, Stori Wide S Jr, dos Santos AF, Mendes RG, et al. Pectus excavatum surgery: sternochondroplasty versus Nuss procedure. Ann Thorac Surg. 2009;88:1773-9.

9. Nuss D, Kelly RE Jr, Croitoru DP, Katz ME. A 10-year review of a minimally invasive technique for the correction of pectus excavatum. J Pediatr Surg 1998;33:545-52.

10. Schalamon J, Pokall S, Windhaber J, Hoellwarth ME. Minimally invasive correction of pectus excavatum in adult patients. J Thorac Cardiovasc Surg. 2006;132: 524-9.

11. Luu TD, Kogon BE, Force SD, Mansour KA, Miller DL. Surgery for recurrent pectus deformities. Ann Thorac Surg. 2009;88:1627-31.

12. Pilegaard HK, Licht PB. Routine use of minimally invasive surgery for pectus excavatum in adults. Ann Thorac Surg. 2008;86:952-6.

13. Kálmán A. Initial results with minimally invasive repair of pectus carinatum. J Thorac Cardiovasc Surg. 2009;138:434-8.

14. Creswick HA, Stacey MW, Kelly RE Jr, Gustin T, Nuss D, Harvey H, et al. Family study of the inheritance of pectus excavatum. J Pediatr Surg. 2006;41: 1699-703.

15. Yoshioka I, Saiki Y, Ichinose A, Takase K, Takahashi S, Ohashi T, et al. Tagged cine magnetic resonance imaging with a finite element model can predict the severity of retrosternal adhesions prior to redo cardiac surgery. J Thorac Cardiovasc Surg. 2009; 137:957-62.

16. Balaras E, Cha KS, Griffith BP, Gammie JS. Treatment of aortic stenosis with aortic valve bypass (apicoaortic conduit) surgery: an assessment using computational modeling. J Thorac Cardiovasc Surg. 2009;137:680-7. 

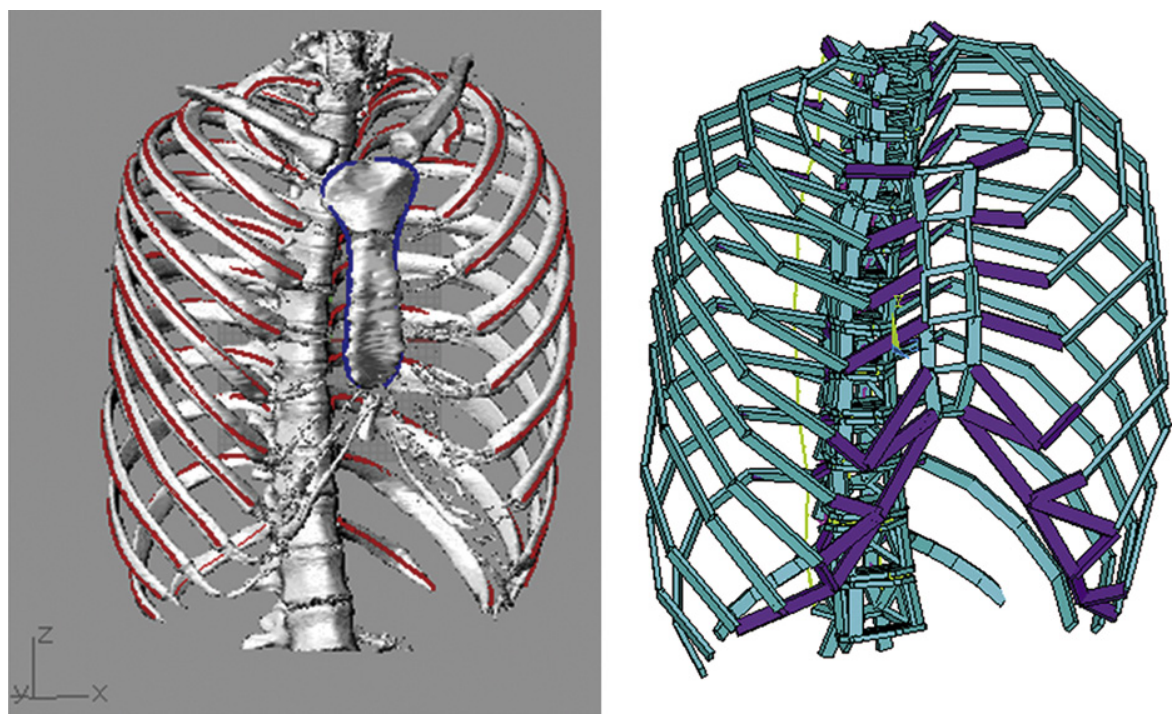

FIGURE E1. Production of biomechanical model with computed tomographic data.
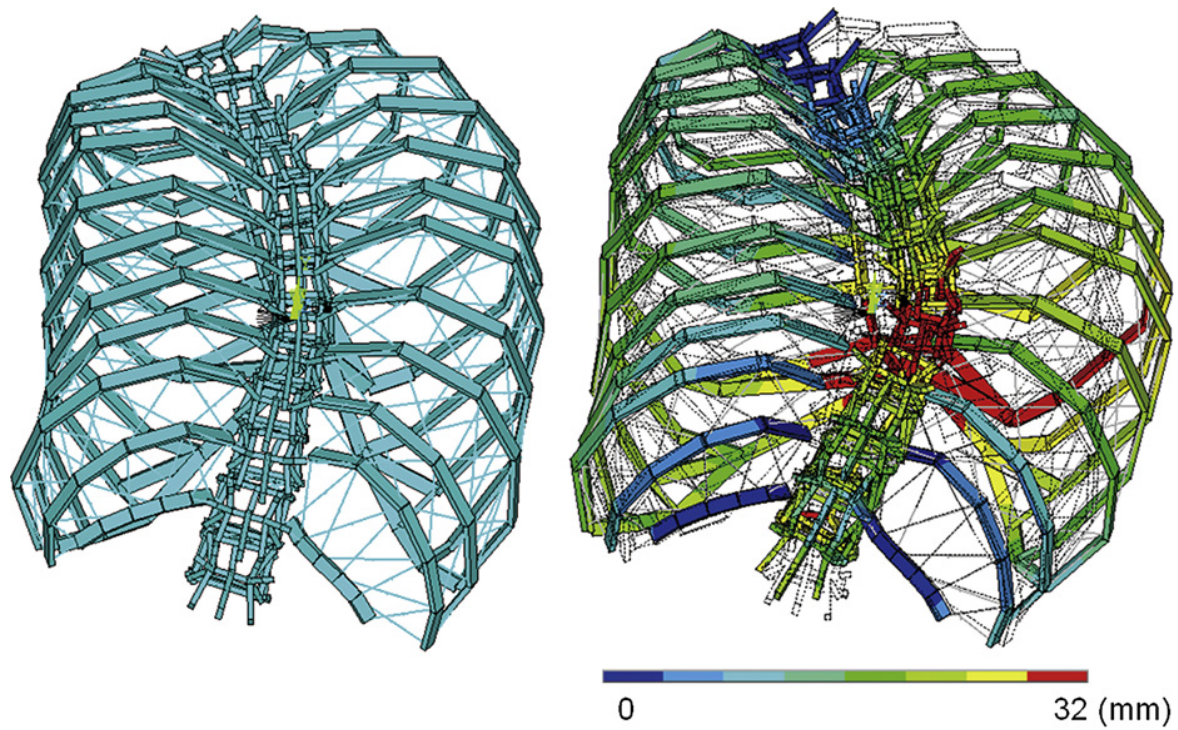

FIGURE E2. Simulation of Nuss procedure for patient with serious scoliosis. 